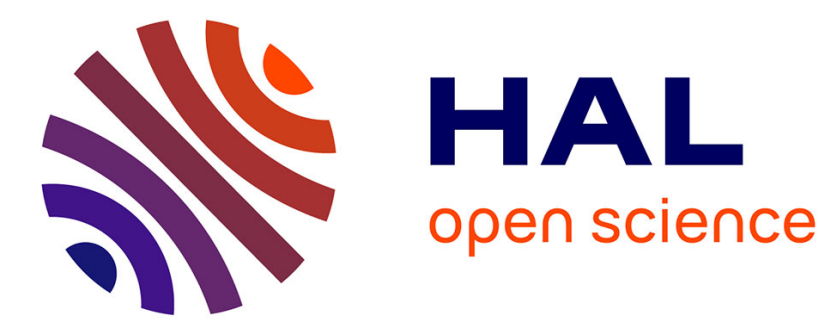

\title{
Axiomatics for oriented connectivity
}

Christian Ronse

\section{To cite this version:}

Christian Ronse. Axiomatics for oriented connectivity. Pattern Recognition Letters, 2014, 47, pp.120128. 10.1016/j.patrec.2014.03.020 . hal-02882869

\section{HAL Id: hal-02882869 \\ https://hal.science/hal-02882869}

Submitted on 21 Jul 2020

HAL is a multi-disciplinary open access archive for the deposit and dissemination of scientific research documents, whether they are published or not. The documents may come from teaching and research institutions in France or abroad, or from public or private research centers.
L'archive ouverte pluridisciplinaire HAL, est destinée au dépôt et à la diffusion de documents scientifiques de niveau recherche, publiés ou non, émanant des établissements d'enseignement et de recherche français ou étrangers, des laboratoires publics ou privés. 


\title{
Axiomatics for oriented connectivity ${ }^{\text {th }}$
}

\author{
Christian Ronse \\ ICube, Université de Strasbourg, CNRS, 300 Boulevard Sébastien Brant, CS 10413, 67412 ILLKIRCH CEDEX, FRANCE
}

\begin{abstract}
Tankyevych et al. (2013) considered, in a directed graph, any set $S$ of vertices where there is some marker vertex $p \in S$ such that for every vertex $x \in S$, there is a directed path from $p$ to $x$ included in $S$; the family of all such sets $S$ was called a semi-connection. Their properties were briefly analysed and compared with connectivity and connected components in undirected graphs.

We give an abstract algebraic formalization of this concept, following the same approach as that of Serra (1988) and Ronse (2008) for the notions of connection and partial connection, which generalize both topological and graph-theoretic connectivity. Here the sets $S$ are unsufficient, one must associate to them their markers $p$; thus in a space $E$ we consider a family $\mathcal{R}$ of ordered pairs $(p, S) \in E \times \mathcal{P}(E)$, where the set $S$ can be "reached" from marker $p$; this family, which we call a reach, must satisfy the three properties of union, transitivity and membership; a fourth point property leads to a full reach. As in (Serra, 1988; Ronse, 2008), we give an equivalent definition in terms of a system of point openings $\left(\gamma_{p}, p \in E\right)$ satisfying some properties. The special case of symmetry, where $S$ does not depend on the choice of the marker $p \in S$, leads to a partial connection or a connection. Some examples are given.

Possible applications of this new theory lie in the analysis of connected structures having an orientation, for instance vascular networks in medical imaging. One can also apply it to geodesic reconstruction and connected filtering.
\end{abstract}

Keywords: oriented path, oriented connectivity, reach, full reach, partial connection, connection

\section{Introduction}

Connectivity is an important issue in morphological image processing, it intervenes in image filtering and segmentation (Ronse and Serra, 2010; Salembier, 2010; Salembier and Wilkinson, 2009; Serra et al., 2010). The notion of a connected object was formalized by Serra (1988) who defined a connection on sets, for which alternate axioms were given in (Ronse, 1998). This concept was generalized by Ronse (2008) who introduced the notion of a partial connection.

Given a non-empty set $E$, a partial connection on $\mathcal{P}(E)$ is a family $C \subseteq \mathcal{P}(E)$ such that $\emptyset \in C$, and for any $\mathcal{B} \subseteq C$ such that $\cap \mathcal{B} \neq \emptyset$, we must have $\cup \mathcal{B} \in C$. When $\{p\} \in C$ for all $p \in E$, we say that $C$ is a connection. Examples of connections include:

- In a topological space, the set of connected sets (i.e., sets that cannot be partitioned by two open sets).

- In a topological space, the set of arc-connected sets (i.e., sets $S$ such that for any $p, q \in S$, there is a continuous map $f:[0,1] \rightarrow S$ with $f(0)=p$ and $f(1)=q)$.

- In an undirected graph, the family of all sets of vertices that are chain-connected (i.e., where any two vertices can be joined by a chain of edges that remains inside the set).

\footnotetext{
This work received funding from the Agence Nationale de la Recherche, contract ANR-2010-BLAN-0205-01.

Email address: cronse@unistra.fr (Christian Ronse)

URL: http://icube-miv.unistra.fr/ (Christian Ronse)
}

These general axioms allowed to define new connectivities, for instance by clustering into a single connected component objects at a small distance from each other (Serra, 1988), or by restricting connectivity to objects having a minimum width (Ronse, 1998). Other examples of connections and partial connections can be found in (Ouzounis and Wilkinson, 2007; Ronse, 2008; Ronse and Serra, 2010).

A partial connection $C$ can be characterized in terms of a system of point openings, that is, a map associating to every $p \in E$ an opening $\gamma_{p}$ on sets, where these openings must satisfy the following two properties:

(TS) For any $p, q \in E$ and $X \in \mathcal{P}(E), q \in \gamma_{p}(X) \Rightarrow \gamma_{q}(X)=$ $\gamma_{p}(X)$.

(M) For any $p \in E$ and $X \in \mathcal{P}(E), p \in X$ or $\gamma_{p}(X)=\emptyset$.

The condition $(\mathrm{M})$ is equivalent to:

$\left(\mathrm{M}^{\prime}\right)$ For any $p \in E$ and $X \in \mathcal{P}(E), p \in \gamma_{p}(X)$ or $\gamma_{p}(X)=\emptyset$.

When $C$ is a connection, a further condition is required:

(P) For any $p \in E, \gamma_{p}(\{p\})=\{p\}$.

It is equivalent to:

$\left(\mathrm{P}^{\prime}\right)$ For any $p \in E$ and $X \in \mathcal{P}(E), p \in X \Rightarrow p \in \gamma_{p}(X)$.

The reason behind the notation (TS), $(\mathrm{M})$ and $(\mathrm{P})$ will be seen in Sections 2 and 3. Then for any $X \in \mathcal{P}(E)$, the $\gamma_{p}(X)$ for $p \in X$ are the connected components of $X$; for a partial connection $C$, 
the connected components of $X$ constitute a partial partition of $X$ (Ronse, 2008), but when $C$ is a connection, they constitute a partition of $X$ (Serra, 1988).

These notions consider connectivity without any orientation. However some structures combine the two; for instance in medical imaging, vascular networks form connected structures (often with further topological properties, such as having neither tunnel nor cavity), but they have also an orientation given by the direction of blood flow. To our knowledge, the first attempt to combine the two was given by Tankyevych et al. (2013) in the framework of directed graphs.

Let $(V, A)$ be a directed graph, where $V$ is the set of vertices and $A$ is the set of arcs (directed/oriented edges); each $\operatorname{arc}$ of $A$ has an origin and a destination, both being vertices of $V$. We assume that the directed graph $(V, A)$ is simple, that is, there are no multiple arcs with the same origin and destination (they do not bring any further connectivity information anyway); thus the unique arc with origin $p$ and destination $q(p, q \in V)$ can be identified by the ordered pair $(p, q)$. Given two vertices $p, q$, a (directed) path from $p$ to $q$ is a sequence $x_{0}, \ldots, x_{n}$ of vertices, where $n \geq 0, p=x_{0}$ and $q=x_{n}$, such that $\left(x_{i-1}, x_{i}\right) \in A$ for $i=1, \ldots, n$. For a set $S$ of vertices, we say that the path $x_{0}, \ldots, x_{n}$ is included in $S$ if each of its vertices belongs to $S$ : $x_{i} \in S(i=0, \ldots, n)$. Given $S \subseteq V$ and a marker vertex $p \in S$, Tankyevych et al. (2013) says that $S$ is semi-connected if for any $q \in S$, there is a path from $p$ to $q$ included in $S$. When $S$ is not semi-connected, one defines the semi-connected components of $S$ as all semi-connected subsets of $S$ that are maximal for inclusion; each such semi-connected subset of $S$ is considered independently from the marker from which its vertices can be joined through directed paths. This structure was called a semi-connection.

A troublesome weakness of this approach is that it considers the semi-connected set $S$ independently from the marker $p$. Indeed, given another $q \in S$, there does not necessarily exist a directed path from $q$ to $p$ that is included in $S$, hence only a subset of $S$ can be reached from $q$ by directed paths included in $S$. Moreover, a union of overlapping semi-connected sets is not necessarily semi-connected. We did not have such a problem with connected sets in a partial connection: here orientation plays no role, so the choice of the marker $p \in S$ is irrelevant, and a union of overlapping connected sets is connected. We will thus consider ordered pairs $(p, S)$, where for any $q \in S$, there is a path from $p$ to $q$ included in $S$. Taking into account the marker $p$ allows giving conditions under which a union of overlapping semi-connected sets is semi-connected: see Definitions 1 and 2 .

Another limitation is that this approach restricts itself to the connectivity in directed graphs; it is an oriented version of graph connectivity. Following the unification of various connectivities under the more general concept of a connection (Serra, 1988; Ronse, 1998) or a partial connection (Ronse, 2008), we can similarly give general axioms for oriented connectivity: given an arbitrary space $E$, we consider a family of ordered pairs $(p, S) \in E \times \mathcal{P}(E)$ satisfying some general properties that are indeed satisfied in the special case of Tankyevych et al. (2013). We will do this in Section 2, and this structure will be called a reach; it is characterized by three axioms called the union, transitivity and membership properties; a fourth point property deals with the connectivity of singletons (as in connections), and it leads to a full reach. We will also characterize a reach or full reach in terms of a system of point openings $\left(\gamma_{p}, p \in E\right)$ satisfying some properties.

In (Serra, 1988; Ronse, 2008), the characterization of a connection or partial connection in terms of a system of point openings satisfying some properties was done globally. Here the correspondence will be built progressively: the union property will give the system of point openings $\left(\gamma_{p}, p \in E\right)$, then the transitivity, membership and point properties will correspond to conditions $(\mathrm{T}),(\mathrm{M})$ and $(\mathrm{P})$ on the $\left(\gamma_{p}, p \in E\right)$. In Section 3 we will consider a further property, symmetry, with a corresponding property $(\mathrm{S})$ on the $\left(\gamma_{p}, p \in E\right)$. We will see that a symmetrical reach is equivalent to a partial connection, and a symmetrical full reach is equivalent to a connection. Indeed, writing (TS) for the conjunction of properties (T) and (S), we get the above conditions.

In Section 4, we will study the complete lattice of reaches, this will allow us to define the reach generated by a family of ordered pairs $(p, S)$ with $p \in S$. Some examples will be given for forests. Section 5 proposes possible applications to the analysis of vascular networks in medical imaging, discusses extensions of the theory towards oriented geodesic reconstruction and connected filtering, then concludes.

\section{Reach axioms}

We first recall some well-known notions (Serra, 1988; Heijmans, 1994; Ronse and Serra, 2010). Let $E$ be a non-empty set. A family $\mathcal{F}$ of subsets of $E$ is closed under union if for any $\mathcal{X} \subseteq \mathcal{F}$, we have $\bigcup \mathcal{X} \in \mathcal{F}$; in particular $\emptyset=\cup \emptyset \in \mathcal{F}$. An equivalent formulation is that for any $X \in \mathcal{P}(E)$, among all $A \in \mathcal{F}$ such that $A \subseteq X$, there is a greatest one (w.r.t. inclusion). An opening on $\mathcal{P}(E)$ is an operator $\gamma: \mathcal{P}(E) \rightarrow \mathcal{P}(E)$ such that for any $X, Y \in \mathcal{P}(E)$, we have $\gamma(Y) \subseteq X \Leftrightarrow \gamma(Y) \subseteq \gamma(X)$. Equivalently, the operator $\gamma$ is anti-extensive, order preserving (isotone) and idempotent. There is a bijection between openings and families of subsets closed under union, under which to the opening $\gamma$ corresponds its invariance domain

$$
\operatorname{lnv}(\gamma)=\{X \in \mathcal{P}(E) \mid \gamma(X)=X\}=\{\gamma(X) \mid X \in \mathcal{P}(E)\},
$$

which is indeed closed under union, while to the family $\mathcal{F}$ closed under union corresponds the opening $\gamma$ defined by

$$
\forall X \in \mathcal{P}(E), \quad \gamma(X)=\bigcup\{A \in \mathcal{F} \mid A \subseteq X\},
$$

in other words, $\gamma(X)$ is the greatest $A \in \mathcal{F}$ such that $A \subseteq X$. This bijection is an isomorphism between the two partially ordered sets of families closed under union and of openings. The least and greatest families closed under union are $\{\emptyset\}$ and $\mathcal{P}(E)$. An intersection of families closed under union will also be closed under union; thus these families form a complete lattice for the inclusion order. Then openings constitute a complete lattice; given a family of opening $\gamma_{i}, i \in I$, there is an opening $\hat{\gamma}$ such 
that $\bigcap_{i \in I} \operatorname{lnv}\left(\gamma_{i}\right)=\operatorname{Inv}(\hat{\gamma})$, and $\hat{\gamma}$ is the infimum of the $\gamma_{i}, i \in$ $I$, in the complete lattice of openings; on the other hand the supremum $\check{\gamma}$ of the $\gamma_{i}$ is their union: $\check{\gamma}(X)=\bigcup_{i \in I} \gamma_{i}(X)$ for all $X \in \mathcal{P}(E)$.

A system of point openings is a map associating to every $p \in$ $E$ an opening $\gamma_{p}$ on $\mathcal{P}(E)$. We write it $\left(\gamma_{p}, p \in E\right)$.

Let us now consider axioms for oriented continuity. We will contemplate a family $\mathcal{R} \subseteq E \times \mathcal{P}(E)$; we say that $S$ is reachable from $p$ when $(p, S) \in \mathcal{R}$. Intuitively, there is some process inside $S$ that allows $p$ to reach all points of $S$. For example in a directed graph $(V, A)$, a natural process is the concatenation of arcs, so $(p, S) \in \mathcal{R}$ if and only if for any $x \in S$ there is a directed path from $p$ to $x$ included in $S$. We first define the following sets:

$$
\begin{aligned}
\mathrm{O}(E) & =\{(p, \emptyset) \mid p \in E\}, \\
\Sigma(E) & =\{(p,\{p\}) \mid p \in E\}, \\
\Psi(E) & =\{(p, S) \in E \times \mathcal{P}(E) \mid p \in S\}, \\
\Phi(E) & =\mathrm{O}(E) \cup \Sigma(E), \\
\Omega(E) & =\mathrm{O}(E) \cup \Psi(E) .
\end{aligned}
$$

We give a first property, which allows a characterization in terms of point openings:

Definition 1. The family $\mathcal{R} \subseteq E \times \mathcal{P}(E)$ satisfies the union property if for any $\mathcal{X} \subseteq \mathcal{P}(E)$ and $p \in E$ such that $(p, S) \in \mathcal{R}$ for every $S \in \mathcal{X}$, we will have $(p, \cup \mathcal{X}) \in \mathcal{R}$. In particular for $\mathcal{X}$ empty, we have $(p, \emptyset) \in \mathcal{R}$ for all $p \in E$, that is, $\mathrm{O}(E) \subseteq \mathcal{R}$.

Proposition 1. There is a bijection between families $\mathcal{R} \subseteq$ $E \times \mathcal{P}(E)$ satisfying the union property and systems of point openings, under which:

- To the family $\mathcal{R}$ satisfying the union property corresponds the system of point openings $\left(\gamma_{p}, p \in E\right)$ defined by:

$$
\forall p \in E, \forall X \in \mathcal{P}(E), \gamma_{p}(X)=\bigcup\{A \subseteq X \mid(p, A) \in \mathcal{R}\},
$$

that is, $\gamma_{p}(X)$ is the greatest $A \subseteq X$ such that $(p, A) \in \mathcal{R}$.

- To the system of point openings $\left(\gamma_{p}, p \in E\right)$ corresponds the family

$$
\begin{aligned}
\mathcal{R} & \left.=\{(p, S)) \mid p \in E, S \in \operatorname{Inv}\left(\gamma_{p}\right)\right\} \\
& =\left\{\left(p, \gamma_{p}(X)\right) \mid p \in E, X \in \mathcal{P}(E)\right\}
\end{aligned}
$$

Proof. Each opening $\gamma_{p}$ corresponds to a family $\mathcal{F}_{p} \subseteq \mathcal{P}(E)$ closed under union. Thus the system $\left(\gamma_{p}, p \in E\right)$ corresponds to the set of ordered pairs $\left(p, \mathcal{F}_{p}\right)$ for all $p \in E$, or to the set

$$
\mathcal{R}=\left\{(p, S) \mid p \in E, S \in \mathcal{F}_{p}\right\} .
$$

In other words, $(p, S) \in \mathcal{R} \Leftrightarrow S \in \mathcal{F}_{p}$. The fact that each $\mathcal{F}_{p}$ is closed under union means that $\mathcal{R}$ satisfies the union property.

Given the family $\mathcal{R}$ satisfying the union property, each $\gamma_{p}$, $p \in E$, is defined by setting for every $X \in \mathcal{P}(E)$ :

$$
\gamma_{p}(X)=\bigcup\left\{A \in \mathcal{F}_{p} \mid A \subseteq X\right\}=\bigcup\{A \subseteq X \mid(p, A) \in \mathcal{R}\},
$$

in fact, the greatest $A \subseteq X$ such that $(p, A) \in \mathcal{R}$.

Given the system of point openings $\left(\gamma_{p}, p \in E\right)$, we have $\mathcal{F}_{p}=\operatorname{Inv}\left(\gamma_{p}\right)=\left\{\gamma_{p}(X) \mid X \in \mathcal{P}(E)\right\}$; combining it with (4), we get (3).

Now we consider the following intuitive idea: if $S$ is reachable from $p$ and $T$ is reachable from $q \in S$, then $S \cup T$ is reachable from $p$. This property holds in the case of directed graphs: (a) for any $x \in S$, we have a path from $p$ to $x$ included in $S \subseteq S \cup T$; (b) for any $x \in T$ we have a path from $q$ to $x$ included in $T$, but then the concatenation of the paths from $p$ to $q$ and from $q$ to $x$ will join $p$ to $x$ and will be included in $S \cup T$; thus from $(\mathrm{a}, \mathrm{b})$ we get that for any $x \in S \cup T$, there is a path from $p$ to $x$ included in $S \cup T$.

Definition 2. The family $\mathcal{R} \subseteq E \times \mathcal{P}(E)$ satisfies the transitivity property if for any $(p, S) \in \mathcal{R}, q \in S$ and $(q, T) \in \mathcal{R}$, we will have $(p, S \cup T) \in \mathcal{R}$.

Proposition 2. Let the family $\mathcal{R} \subseteq E \times \mathcal{P}(E)$ satisfy the union property, and let $\left(\gamma_{p}, p \in E\right)$ be the corresponding system of point openings. Then $\mathcal{R}$ satisfies the transitivity property if and only if $\left(\gamma_{p}, p \in E\right)$ satisfies the following:

(T) For any $p, q \in E$ and $X \in \mathcal{P}(E), q \in \gamma_{p}(X) \Rightarrow \gamma_{q}(X) \subseteq$ $\gamma_{p}(X)$

Proof. Suppose that $\mathcal{R}$ satisfies the transitivity property. Let $p, q \in E$ and $X \in \mathcal{P}(E)$ such that $q \in \gamma_{p}(X)$. By (3) we have $\left(p, \gamma_{p}(X)\right),\left(q, \gamma_{q}(X)\right) \in \mathcal{R}$. The transitivity property implies that $\left(p, \gamma_{p}(X) \cup \gamma_{q}(X)\right) \in \mathcal{R}$, so by (3) again, we have $\gamma_{p}(X) \cup \gamma_{q}(X) \in$ $\operatorname{Inv}\left(\gamma_{p}\right)$; as $\gamma_{p}(X) \subseteq \gamma_{p}(X) \cup \gamma_{q}(X) \subseteq X$, this means that $\gamma_{p}(X) \cup$ $\gamma_{q}(X)=\gamma_{p}(X)$, that is, $\gamma_{q}(X) \subseteq \gamma_{p}(X)$. So (T) holds.

Suppose now that (T) holds. Let $(p, S) \in \mathcal{R}, q \in S$ and $(q, T) \in \mathcal{R}$. By (3), $S \in \operatorname{lnv}\left(\gamma_{p}\right)$ and $T \in \operatorname{Inv}\left(\gamma_{q}\right)$. Thus $S=$ $\gamma_{p}(S) \subseteq \gamma_{p}(S \cup T), q \in \gamma_{p}(S \cup T)$ and $T=\gamma_{q}(T) \subseteq \gamma_{q}(S \cup T)$. Applying (T) with $X=S \cup T$, we get $\gamma_{q}(S \cup T) \subseteq \gamma_{p}(S \cup T)$. Hence $S, T \subseteq \gamma_{p}(S \cup T)$, so $S \cup T \subseteq \gamma_{p}(S \cup T)$, which means that $S \cup T \in \operatorname{Inv}\left(\gamma_{p}\right)$; then (3) gives $(p, S \cup T) \in \mathcal{R}$. Therefore the transitivity property is satisfied.

Our third property concerns the relation between the marker $p$ and the set $S$ reachable from it: Tankyevych et al. (2013) requires a path from $p$ to any point in $S$, this path being included in $S$; hence $p \in S$. When $p$ neighbours $S$, we must consider that $S \cup\{p\}$, and not $S$, is reached from $p$. We postulate thus that the marker always belongs to the reachable set, except for the empty set, which can always be reached.

Definition 3. The family $\mathcal{R} \subseteq E \times \mathcal{P}(E)$ satisfies the membership property if $\mathcal{R} \subseteq \Omega(E)$, that is, for any $(p, S) \in \mathcal{R}$, either $S=\emptyset$ or $p \in S$.

Proposition 3. Let the family $\mathcal{R} \subseteq E \times \mathcal{P}(E)$ satisfy the union property, and let $\left(\gamma_{p}, p \in E\right)$ be the corresponding system of point openings. Then $\mathcal{R}$ satisfies the membership property if and only if $\left(\gamma_{p}, p \in E\right)$ satisfies the following:

(M) For any $p \in E$ and $X \in \mathcal{P}(E), p \in X$ or $\gamma_{p}(X)=\emptyset$.

It is equivalent to: 
$\left(\mathrm{M}^{\prime}\right)$ For any $p \in E$ and $X \in \mathcal{P}(E), p \in \gamma_{p}(X)$ or $\gamma_{p}(X)=\emptyset$.

Proof. For $(p, S) \in E \times \mathcal{P}(E)$, by (3) we have $(p, S) \in \mathcal{R}$ if and only if there is some $X \in \mathcal{P}(E)$ such that $S=\gamma_{p}(X)$. The membership property states that either $S=\emptyset$ or $p \in S$, in other words $\gamma_{p}(X)=\emptyset$ or $p \in \gamma_{p}(X)$, that is $\left(\mathbf{M}^{\prime}\right)$.

Now $(\mathrm{M})$ and $\left(\mathrm{M}^{\prime}\right)$ correspond to properties $(\mathrm{C} 1 \mathrm{a})$ and $(\mathrm{C} 1 \mathrm{~b})$ of Ronse (2008), the equivalence between them was shown in Lemma 19 of that paper.

In order to have the analogy with a connection, where singletons are connected, we finally consider the property that a point can be reached from itself:

Definition 4. The family $\mathcal{R} \subseteq E \times \mathcal{P}(E)$ satisfies the point property if $\Sigma(E) \subseteq \mathcal{R}$, that is, for any $p \in E$ we have $(p,\{p\}) \in \mathcal{R}$.

Proposition 4. Let the family $\mathcal{R} \subseteq E \times \mathcal{P}(E)$ satisfy the union property, and let $\left(\gamma_{p}, p \in E\right)$ be the corresponding system of point openings. Then $\mathcal{R}$ satisfies the point property if and only if $\left(\gamma_{p}, p \in E\right)$ satisfies the following:

(P) For any $p \in E, \gamma_{p}(\{p\})=\{p\}$.

It is equivalent to:

$\left(\mathrm{P}^{\prime}\right)$ For any $p \in E$ and $X \in \mathcal{P}(E), p \in X \Rightarrow p \in \gamma_{p}(X)$.

Proof. For $p \in E$, by (3) we have:

$$
(p,\{p\}) \in \mathcal{R} \Longleftrightarrow\{p\} \in \operatorname{Inv}\left(\gamma_{p}\right) \Longleftrightarrow \gamma_{p}(\{p\})=\{p\} ;
$$

Thus $\mathcal{R}$ satisfies the point property if and only if $(\mathrm{P})$ holds.

Now $(\mathrm{P})$ and $\left(\mathrm{P}^{\prime}\right)$ correspond to properties $(\mathrm{COa})$ and $(\mathrm{COb})$ of Ronse (2008), the equivalence between them was shown in Lemma 19 of that paper.

We do not require any further property in order to define the notion of a reach:

Definition 5. A reach on $E$ is a family $\mathcal{R} \subseteq E \times \mathcal{P}(E)$ satisfying the union, transitivity and membership properties. If it satisfies also the point property, we call it a full reach.

From Propositions 1, 2, 3 and 4 we deduce:

Corollary 5. There is a bijection between reaches and systems of point openings satisfying both $(\mathrm{T})$ and $(\mathrm{M}) /\left(\mathrm{M}^{\prime}\right)$. A reach $\mathcal{R}$ and a system of point openings $\left(\gamma_{p}, p \in E\right)$ correspond to each other by $(2,3)$. The reach is full if and only if the system of point openings satisfies $(\mathrm{P}) /\left(\mathrm{P}^{\prime}\right)$.

We will see in Section 3 that for a partial connection $C$, the set of all $(p, S)$, where $p \in S \in C$ or $S=\emptyset$, is a reach; for a connection, this gives a full reach. In a directed graph $(V, A)$, the set of ordered pairs $(p, S) \in \Omega(V)$ such that $S=\emptyset$ or for any $q \in S$ there is a directed path from $p$ to $q$ included in $S$, constitutes a full reach; we call it the Tankyevych reach and write it $\mathcal{T}_{(V, A)}$; here for $p \in X, \gamma_{p}(X)$ is the set of all $x \in X$ such that there is a directed path from $p$ to $x$ included in $X$.
Let us give other examples. Our first ones rely on a quasiorder on $E$, that is, a reflexive and transitive binary relation $\sqsubseteq$ on $E$; the inverse relation $\sqsupseteq$ is also a quasi-order. Now

$$
\mathcal{H}_{\sqsubseteq}(E)=\mathrm{O}(E) \cup\{(p, S) \in \Psi(E) \mid \forall q \in S, p \sqsubseteq q\}
$$

is a full reach, we call it the $\sqsubseteq$-reach. For instance, given a map $g: E \rightarrow T$ and a quasi-order $\leq$ on $T$, we get a quasi-order $\sqsubseteq$ on $E$ defined by $p \sqsubseteq q \Leftrightarrow g(p) \preceq g(q)$; if $g$ is a numerical function defining a grey-level image on $E$ and $\leq$ is the numerical order on $T$, then $\sqsubseteq$ compares points of $E$ according to their values in the image, and for $p \in X \subseteq E, \gamma_{p}(X)=\{x \in X \mid g(p) \leq g(x)\}$, the thresholding of $X$ above level $g(p)$.

When $E$ is a topological space with a quasi-order $\sqsubseteq$, we get an ordered variant of arc-connectivity. Let $\mathcal{A}_{\sqsubseteq}(E)$ be made of $\mathrm{O}(E)$ and of all $(p, S) \in \Psi(E)$ such that for any $q \in S$, there is a continuous map $f:[0,1] \rightarrow S$ with $f(0)=p, f(1)=q$, and for $0 \leq s \leq t \leq 1$, we have $f(s) \sqsubseteq f(t)$; in other words, either $S=\emptyset$, or all points of $S$ can be reached from $p$ by an arc in $S$ which is increasing for $\sqsubseteq$. Then $\mathcal{A}_{\sqsubseteq}(E)$ is a full reach.

Given a simple directed or undirected graph $(V, A)$ with a quasi-order $\sqsubseteq$ on $V$, we define in an analogous way the full reach $\mathcal{A}_{(V, \subseteq, A)}$, included in $\mathcal{T}_{(V, A)}$. Again we take ordered pairs $(p, S) \in \Omega(V)$ such that $S=\emptyset$ or for any $q \in S$ there is a directed path from $p$ to $q$ included in $S$, but we require that this path $p=x_{0}, \ldots, x_{n}=q$ is increasing for $\sqsubseteq: x_{0} \sqsubseteq \cdots \sqsubseteq x_{n}$. For instance, let $V$ be a digital set of pixels, $A$ the set of arcs corresponding to ordered pairs of adjacent pixels, and $T$ a set of numerical values ordered by $\leq$; given a numerical function $g: V \rightarrow T$, we define the quasi-order $\sqsubseteq$ on $V$ by $p \sqsubseteq q \Leftrightarrow g(p) \leq g(q)$. Thus $\mathcal{A}_{(V, \sqsubseteq, A)}$ will consist of all $(p, S)$ such that $S=\emptyset$ or for any $q \in S$ there is in $S$ a path from $p$ to $q$ where the function $g$ is increasing on the pixels; for $\mathcal{A}_{(V, A, \sqsupseteq)}$, the path must be decreasing for $g$.

We can take a dual view and define a full reach on the set of arcs. Here we exchange the role of vertices and arcs in the above discussion; a path will be a sequence of $\operatorname{arcs} a_{1}, \ldots, a_{n}$, such that for $i=2, \ldots, n$, the destination of $a_{i-1}$ is the origin of $a_{i}$. Note that the graph is no more assumed to be simple. We can then define the dual Tankyevych reach $\mathcal{T}_{(A, V)}^{*}$, where from a marker arc we can reach a set of arcs thanks to paths starting in the marker and ending in the arcs of the set. If we have a quasi-order $\unlhd$ on $A$, we define similarly the full reach $\mathcal{A}_{(A, \triangleleft, V)}^{*}$, included in $\mathcal{T}_{(A, V)}^{*}$; here the path $a_{1}, \ldots, a_{n}$ must be increasing; for a numerical function $g: A \rightarrow T$, we get the quasi-order $\unlhd$ on $A$ by $a \unlhd a^{\prime} \Leftrightarrow g(a) \leq g\left(a^{\prime}\right)$; thus for $\mathcal{A}_{(A, \unlhd, V)}^{*}$, arcs are reached through a the path on which the function $g$ is increasing on the arcs; for $\mathcal{A}_{(A, \unrhd, V)}^{*}$, the function must decrease on the path. Paths with decreasing values on the arcs are often considered in relation to watersheds (Bertrand et al., 2010; Cousty et al., 2014), thus the full reach $\mathcal{A}_{(A, \unrhd, V)}^{*}$ is relevant here.

Our final example deals with rooted forests, we explain it through genealogy and inheritance. A woman receives some money. Then the following rule can be repeated a finite number of times:

- any woman having some money can either keep it for herself, or share it equally between her daughters. 
If we model the genealogy of women as a union of disjoint rooted trees, with women as vertices and an arc (directed edge) from mother to daughter (they are thus parent and child vertex), then the initial woman $p$ and the set $S$ of all women having received some money satisfy the following:

1. $p \in S$.

2. For any $q \in S \backslash\{p\}, q$ is a descendant of $p$, and the parent of $q$ belongs to $S$.

3. For any $q \in S$, if one child of $q$ belongs to $S$, then all children of $q$ belong to $S$.

The set of all pairs $(p, S)$ satisfying items 1, 2 and 3, together with $\mathrm{O}(E)$, constitutes a full reach. We call it the inheritance reach. We illustrate it in Figure 1. Note that a set $S$ of vertices is connected in the underlying undirected forest if and only if there is some vertex $p$ for which items 1 and 2 hold (it is in fact a particular case of the Tankyevych reach); however item 3 is not necessarily satisfied.
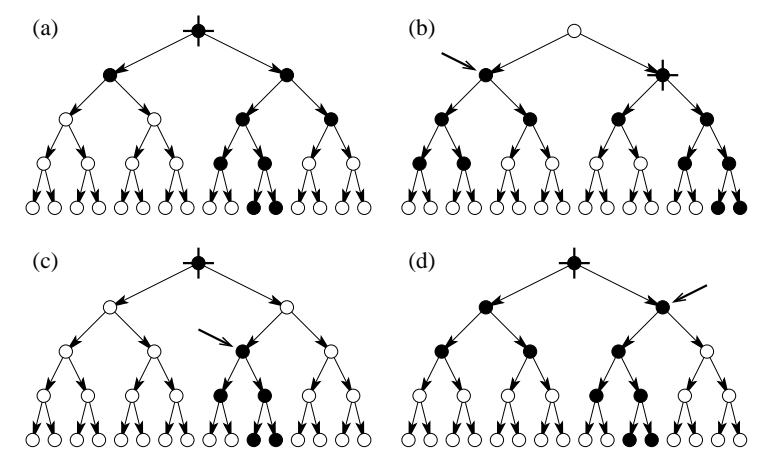

Figure 1: We consider a binary rooted tree (with the root at the top). In each example (a,b,c,d), the marker $p$ is indicated by a cross, $S$ is the set of vertices shown as filled disks, while vertices not in $S$ are shown as hollow disks. In (a), $(p, S)$ belongs to the inheritance reach; in (b,c,d) it does not, and an arrow indicates a vertex $q \in S$ that does not satisfy some rule: in (b), $q$ is not a descendant of $p$; in (c), $q$ is a descendant of $p$, but its parent does not belong to $S$; in (d), $q$ has one child in $S$ but the other not in $S$.

In Proposition 18 of (Ronse, 2008), we showed that for $C \subseteq$ $\mathcal{P}(E), C$ is a partial connection if and only if by adding to $C$ all singletons in $E$, we get a connection. We give here an analogous result:

Proposition 6. A family $\mathcal{R} \subseteq E \times \mathcal{P}(E)$ is a reach if and only if $\mathcal{R} \cup \Sigma(E)$ is a full reach; then $\mathcal{R} \cup \Sigma(E)$ is the least full reach containing $\mathcal{R}$. Given $\left(\gamma_{p}, p \in E\right)$ and $\left(\hat{\gamma}_{p}, p \in E\right)$ the two systems of point openings corresponding to $\mathcal{R}$ and $\mathcal{R} \cup \Sigma(E)$ respectively, we have for any $p \in E$ and $X \in \mathcal{P}(E)$ :

$$
\hat{\gamma}_{p}(X)= \begin{cases}\{p\} & \text { if } p \in X \text { and } \gamma_{p}(X)=\emptyset \\ \gamma_{p}(X) & \text { otherwise } .\end{cases}
$$

Proof. Suppose that $\mathcal{R}$ is a reach. Let $\mathcal{X} \subseteq \mathcal{P}(E)$ and $p \in E$ such that $(p, S) \in \mathcal{R} \cup \Sigma(E)$ for every $S \in \mathcal{X}$; set $\mathcal{Y}=\{S \in$ $\mathcal{X} \mid(p, S) \in \mathcal{R}\}$. By the union property on $\mathcal{R},(p, \cup \mathcal{Y}) \in \mathcal{R}$. If $\cup \mathcal{Y}=\bigcup \mathcal{X}$, then $(p, \cup \mathcal{X}) \in \mathcal{R}$. Assume now that $\bigcup \mathcal{Y} \neq \cup \mathcal{X}$; then $\mathcal{Y} \subset \mathcal{X}$, and for any $S \in \mathcal{X} \backslash \mathcal{Y},(p, S) \notin \mathcal{R}$, so $(p, S) \in \Sigma(E)$, which means that $S=\{p\}$; thus $\bigcup \mathcal{X}=\bigcup \mathcal{Y} \cup\{p\}$; as $\bigcup \mathcal{Y} \neq$ $\cup \mathcal{X}, p \notin \cup \mathcal{Y}$, so $p \notin S$ for all $S \in \mathcal{Y}$; by the membership property on $\mathcal{R}, \mathcal{Y}=\emptyset$ or $\mathcal{Y}=\{\emptyset\}$, so $\cup \mathcal{Y}=\emptyset$; therefore $\cup \mathcal{X}=$ $\cup \mathcal{Y} \cup\{p\}=\{p\}$ and $(p, \cup \mathcal{X})=(p,\{p\}) \in \Sigma(E)$. We have thus shown that $(p, \cup \mathcal{X}) \in \mathcal{R} \cup \Sigma(E)$ in any case, so $\mathcal{R} \cup \Sigma(E)$ satisfies the union property.

Let $(p, S) \in \mathcal{R} \cup \Sigma(E), q \in S$ and $(q, T) \in \mathcal{R} \cup \Sigma(E)$. If $(q, T) \in \Sigma(E)$, then $T=\{q\}$, and as $q \in S, S \cup T=S$; similarly, if $T=\emptyset$, then $S \cup T=S$; in both cases, $(p, S \cup T)=(p, S) \in$ $\mathcal{R} \cup \Sigma(E)$. Assume now that $\emptyset \neq T \neq\{q\}$; then $(q, T) \in \mathcal{R}$, and by the membership property on $\mathcal{R}, q \in T$. If $(p, S) \in \Sigma(E)$, then $S=\{p\}$, and as $q \in S, p=q$; now $q \in T$, so $S \cup T=$ $\{p\} \cup T=\{q\} \cup T=T$, so $(p, S \cup T)=(q, T) \in \mathcal{R} \cup \Sigma(E)$. If $(p, S) \notin \Sigma(E)$, then $(p, S) \in \mathcal{R}$, and as $q \in S$ and $(q, T) \in \mathcal{R}$, we get $(p, S \cup T) \in \mathcal{R}$ by the transitivity property on $\mathcal{R}$. We have thus shown that $(p, S \cup T) \in \mathcal{R} \cup \Sigma(E)$ in any case, so $\mathcal{R} \cup \Sigma(E)$ satisfies the transitivity property.

Let $(p, S) \in \mathcal{R} \cup \Sigma(E)$. If $(p, S) \in \mathcal{R}$, then $S=\emptyset$ or $p \in S$ by the membership property on $\mathcal{R}$; if $(p, S) \notin \mathcal{R}$, then $(p, S) \in$ $\Sigma(E)$, which means that $S=\{p\}$, so $p \in S$. Therefore $\mathcal{R} \cup \Sigma(E)$ satisfies the membership property.

Obviously $\mathcal{R} \cup \Sigma(E)$ satisfies the point property. We have thus shown that $\mathcal{R} \cup \Sigma(E)$ is a full reach.

Conversely, suppose that $\mathcal{R} \cup \Sigma(E)$ is a full reach. Let $X \subseteq$ $\mathcal{P}(E)$ and $p \in E$ such that $(p, S) \in \mathcal{R}$ for every $S \in \mathcal{X}$. By the union property on $\mathcal{R} \cup \Sigma(E),(p, \cup \mathcal{X}) \in \mathcal{R} \cup \Sigma(E)$. Thus $(p, \cup \mathcal{X}) \in \mathcal{R}$ or $(p, \cup \mathcal{X}) \in \Sigma(E)$; in the latter case, $\cup \mathcal{X}=$ $\{p\}$, hence $\{p\} \in \mathcal{X}$ and $(p, \cup \mathcal{X})=(p,\{p\}) \in \mathcal{R}$. Therefore $(p, \cup \mathcal{X}) \in \mathcal{R}$ in any case, and $\mathcal{R}$ satisfies the union property.

Let $(p, S) \in \mathcal{R}, q \in S$ and $(q, T) \in \mathcal{R}$. By the transitivity property on $\mathcal{R} \cup \Sigma(E),(p, S \cup T) \in \mathcal{R} \cup \Sigma(E)$. Thus $(p, S \cup T) \in$ $\mathcal{R}$ or $(p, S \cup T) \in \Sigma(E)$; in the latter case, $S \cup T=\{p\}$; as $q \in S, S \neq \emptyset$, so $S=\{p\}=S \cup T$, and $(p, S \cup T)=(p, S) \in$ $\mathcal{R}$. Therefore $(p, S \cup T) \in \mathcal{R}$ in any case, and $\mathcal{R}$ satisfies the transitivity property.

Let $(p, S) \in \mathcal{R}$. By the membership property on $\mathcal{R} \cup \Sigma(E)$, $S=\emptyset$ or $p \in S$. Therefore $\mathcal{R}$ satisfies the membership property. We have shown that $\mathcal{R}$ is a reach.

Every full reach containing $\mathcal{R}$ must also contain $\Sigma(E)$, hence $\mathcal{R} \cup \Sigma(E)$ is the least full reach containing $\mathcal{R}$.

By (2), we have for $p \in E$ and $X \in \mathcal{P}(E)$ :

$$
\begin{gathered}
\hat{\gamma}_{p}(X)=\bigcup\{A \subseteq X \mid(p, A) \in \mathcal{R} \cup \Sigma(E)\} \\
=\bigcup\{A \subseteq X \mid(p, A) \in \mathcal{R}\} \cup \bigcup\{A \subseteq X \mid(p, A) \in \Sigma(E)\} \\
=\gamma_{p}(X) \cup \bigcup\{A \subseteq X \mid A=\{p\}\}= \begin{cases}\gamma_{p}(X) & \text { if } p \notin X, \\
\gamma_{p}(X) \cup\{p\} & \text { if } p \in X .\end{cases}
\end{gathered}
$$

Now for $p \in X,\left(\mathrm{M}^{\prime}\right)$ gives either $\gamma_{p}(X)=\emptyset$, so $\hat{\gamma}_{p}(X)=\{p\}$, or $p \in \gamma_{p}(X)$, so $\hat{\gamma}_{p}(X)=\gamma_{p}(X)$.

In a directed graph $(V, A)$, Tankyevych et al. (2013) defined the semi-connected components of a subset $X$ of $V$ as the $\gamma_{p}(X)$, $p \in X$; since $\mathcal{T}_{(V, A)}$ is a full reach, these components are nonempty and cover $X$, thanks to $\left(\mathrm{P}^{\prime}\right)$. In a reach $\mathcal{R}$ that is not full, we must eliminate the empty set from the components, as we 
did in (Ronse, 2008) for partial connections; so we consider all non-empty $\gamma_{p}(X)$, that is, the $\gamma_{p}(X)$ such that $p \in \gamma_{p}(X)$, cf. $\left(\mathbf{M}^{\prime}\right)$; here the $\gamma_{p}(X), p \in X$, do not necessarily cover $X$. One could also eliminate the components that are not maximal for inclusion: we do not consider $\gamma_{q}(X)$ when $\gamma_{q}(X) \subset \gamma_{p}(X)$ (in this case we necessarily have $q \in \gamma_{p}(X)$ by the membership property); in particular if $V$ is finite, every component is included in a maximal component.

Several properties of connected components (in a partial connection) are not satisfied by semi-connected components. The set of all maximal $\gamma_{p}(X)$ does not necessarily constitute a partial partition (Ronse, 2008), nor even a hierarchy (Serra, 2011): we can have $\gamma_{p}(X) \cap \gamma_{q}(X) \neq \emptyset$ with both $\gamma_{p}(X) \nsubseteq \gamma_{q}(X)$ and $\gamma_{q}(X) \nsubseteq \gamma_{p}(X)$; furthermore $\gamma_{p}(X) \cup \gamma_{q}(X)$ will not necessarily be semi-connected. For example take $V=\{a, b, c, d\}$ and $A=\{(a, c),(b, c),(c, d)\}$; then in $\mathcal{T}_{(V, A)}$ we have $\gamma_{a}(V)=\{a, c, d\}$ and $\gamma_{b}(V)=\{b, c, d\}$; they are maximal semi-connected components, since the only greater set is $V$, which is not semiconnected (there is no directed path from $a$ to $b$ or from $b$ to $a)$; now $\gamma_{a}(V) \cap \gamma_{b}(V) \neq \emptyset, \gamma_{a}(V) \nsubseteq \gamma_{b}(V), \gamma_{b}(V) \nsubseteq \gamma_{a}(V)$ and $\gamma_{a}(V) \cup \gamma_{b}(V)=V$, which is not semi-connected. Other examples are given in Perret et al. (2014).

\section{Symmetry and links with connections}

In a directed graph $(V, A)$, a set $S$ of vertices is strongly connected if for any $p, q \in S$ there is a directed path from $p$ to $q$ and one from $q$ to $p$. It is easily seen (by concatenating paths) that the set of strongly connected subsets of $V$ constitutes a connection. In the Tankyevych reach $\mathcal{T}_{(V, A)}$, a strongly connected set $S$ satisfies $(p, S) \in \mathcal{T}_{(V, A)}$ for all $p \in S$. We will take this property as the basis for constructing a partial connection from a reach $\mathcal{R}$, the symmetrical part $\operatorname{Sym}(\mathcal{R})$.

Definition 6. In a reach $\mathcal{R}$, the symmetrical part is the family

$$
\operatorname{Sym}(\mathcal{R})=\{S \in \mathcal{P}(E) \mid \forall q \in S,(q, S) \in \mathcal{R}\} .
$$

We say that $\mathcal{R}$ is symmetrical iffor all $(p, S) \in \mathcal{R}, S \in \operatorname{Sym}(\mathcal{R})$.

Proposition 7. Let $\mathcal{R}$ be a reach, and let $\left(\gamma_{p}, p \in E\right)$ be the corresponding system of point openings. Then

$$
\operatorname{Sym}(\mathcal{R})=\left\{S \in \mathcal{P}(E) \mid \forall q \in S, S=\gamma_{q}(S)\right\} .
$$

In particular, $\emptyset \in \operatorname{Sym}(\mathcal{R})$. Furthermore, $\mathcal{R}$ is symmetrical if and only if $\left(\gamma_{p}, p \in E\right)$ satisfies the following:

(S) For any $p, q \in E$ and $X \in \mathcal{P}(E), q \in \gamma_{p}(X) \Rightarrow \gamma_{q}(X) \supseteq$ $\gamma_{p}(X)$.

Proof. Let $S \in \mathcal{P}(E)$. Then $S \in \operatorname{Sym}(\mathcal{R})$ iff for any $q \in S$, $(q, S) \in \mathcal{R}$, which means by (3) that $S \in \operatorname{lnv}\left(\gamma_{q}\right)$, in other words $S=\gamma_{q}(S)$. Hence (7) holds. The condition of $(6,7)$ is trivially satisfied for $S=\emptyset$, thus $\emptyset \in \operatorname{Sym}(\mathcal{R})$.

Suppose that $\mathcal{R}$ is symmetrical. Let $p, q \in E$ and $X \in \mathcal{P}(E)$ such that $q \in \gamma_{p}(X)$. By (3) we have $\left(p, \gamma_{p}(X)\right) \in \mathcal{R}$. Thus $\gamma_{p}(X) \in \operatorname{Sym}(\mathcal{R})$, so (7) gives $\gamma_{p}(X)=\gamma_{q}\left(\gamma_{p}(X)\right)$; since $\gamma_{p}, \gamma_{q}$ are openings, we have $\gamma_{p}(X) \subseteq X$, so $\gamma_{q}\left(\gamma_{p}(X)\right) \subseteq \gamma_{q}(X)$. Hence $\gamma_{p}(X)=\gamma_{q}\left(\gamma_{p}(X)\right) \subseteq \gamma_{q}(X)$ and $(\mathrm{S})$ holds.

Suppose now that (S) holds. Let $(p, S) \in \mathcal{R}$; by (3) we have $S \in \operatorname{Inv}\left(\gamma_{p}\right)$, that is, $S=\gamma_{p}(S)$. Now for any $q \in S$, we have $q \in \gamma_{p}(S)$, so applying (S) with $X=S$, we get $\gamma_{q}(S) \supseteq$ $\gamma_{p}(S)$; but (T) gives $\gamma_{q}(S) \subseteq \gamma_{p}(S)$, hence $\gamma_{q}(S)=\gamma_{p}(S)=S$. Therefore $S \in \operatorname{Sym}(\mathcal{R})$ by (7); as this holds for all $(p, S) \in \mathcal{R}$, $\mathcal{R}$ is symmetrical.

We get then the following generalization of strongly connected sets in a directed graph:

Proposition 8. The symmetrical part of a reach is a partial connection. The symmetrical part of a full reach is a connection.

Proof. Let $\mathcal{R}$ be a reach. We have $\emptyset \in \operatorname{Sym}(\mathcal{R})$. Let $X \subseteq$ $\operatorname{Sym}(\mathcal{R})$ with $\cap \mathcal{X} \neq \emptyset$, and let $x \in \cap \mathcal{X}$. For any $q \in \cup \mathcal{X}$ there is some $S \in \mathcal{X}$ such that $q \in S$; as $S \in \operatorname{Sym}(\mathcal{R}),(q, S) \in \mathcal{R}$. For any $T \in \mathcal{X} \backslash\{S\}$, as $x \in T \in \operatorname{Sym}(\mathcal{R}),(x, T) \in \mathcal{R}$; as $(q, S) \in \mathcal{R}, x \in S$ and $(x, T) \in \mathcal{R}$, the transitivity property gives $(q, S \cup T) \in \mathcal{R}$. Now $\cup_{T \in \mathcal{X} \backslash\{S\}}(S \cup T)=\bigcup \mathcal{X}$, hence the union property gives $(q, \cup \mathcal{X})=\left(q, \cup_{T \in \mathcal{X} \backslash\{S\}}(S \cup T)\right) \in \mathcal{R}$. As $(q, \cup \mathcal{X}) \in \mathcal{R}$ for all $q \in \cup \mathcal{X}, \cup \mathcal{X} \in \operatorname{Sym}(\mathcal{R})$. Therefore $\operatorname{Sym}(\mathcal{R})$ is a partial connection.

If $\mathcal{R}$ is full, then for any $p \in E$ we have $(p,\{p\}) \in \mathcal{R}$. For any $q \in\{p\}, q=p$, so $(q,\{p\}) \in \mathcal{R}$. Thus $\{p\} \in \operatorname{Sym}(\mathcal{R})$ and $\operatorname{Sym}(\mathcal{R})$ is a connection.

Let us consider the symmetrical part for some examples of reaches given in Section 2. The symmetrical part of the Tankyevych reach $\mathcal{T}_{(V, A)}$ consists of all strongly connected subsets of $V$. Now consider a map $g: E \rightarrow T$ with a partial order $\leq$ on $T$, and define the quasi-order $\sqsubseteq$ on $E$ by $p \sqsubseteq q \Leftrightarrow g(p) \leq$ $g(q)$. The symmetrical part of $\mathcal{H}_{\sqsubseteq}(E)$ consists of all $S \subseteq E$ such that $g$ takes a constant value on $S$. When $E$ is a topological space, $\operatorname{Sym}\left(\mathcal{A}_{\sqsubseteq}(E)\right)$ consists of all arc-connected $S \subseteq E$ on which $g$ takes a constant value. Finally, for $E=V$ in a graph $(V, A), \operatorname{Sym}\left(\mathcal{A}_{(V, \subseteq, A)}\right)$ is the set of strongly connected $S \subseteq V$ on which $g$ takes a constant value. All three are obviously connections.

Comparing (T) and (S), they differ only in the sense of the inclusion relation between $\gamma_{q}(X)$ and $\gamma_{p}(X)$. Thus their conjunction takes the following form:

(TS) For any $p, q \in E$ and $X \in \mathcal{P}(E), q \in \gamma_{p}(X) \Rightarrow \gamma_{q}(X)=$ $\gamma_{p}(X)$.

Now (TS) and (M) / ( $\left.\mathbf{M}^{\prime}\right)$, the axioms satisfied by the system of point openings of a symmetrical reach, are precisely those given for the system of point openings of a partial connection; the supplementary axioms $(\mathrm{P}) /\left(\mathrm{P}^{\prime}\right)$ for a full reach are exactly those added for a connection. We have thus a natural bijection between symmetrical reaches and partial connections, and in particular between symmetrical full reaches and connections:

Proposition 9. Let $C \subseteq \mathcal{P}(E)$ and let

$\mathcal{R}=(E \times C) \cap \Omega(E)=\{(p, S) \mid p \in E, S \in C, p \in S$ or $S=\emptyset\}$. 
Then $C$ is a partial connection if and only if $\mathcal{R}$ is a reach (which is thus symmetrical), and $C$ is a connection if and only if $\mathcal{R}$ is a full reach.

Proof. If $\mathcal{R}$ is a reach, then it is necessarily symmetrical, since for $(p, S) \in \mathcal{R}$ we have $(q, S) \in \mathcal{R}$ for all $q \in S$. Also we have $C=\operatorname{Sym}(\mathcal{R})$. Hence by Proposition 8 , if $\mathcal{R}$ is a reach, then $C$ is a partial connection, and if $\mathcal{R}$ is a full reach, then $C$ is a connection.

Now suppose that $C$ is a partial connection. Since $\mathcal{R} \subseteq \Omega(E)$, $\mathcal{R}$ satisfies the membership property.

Let $\mathcal{X} \subseteq \mathcal{P}(E)$ such that $(p, S) \in \mathcal{R}$ for every $S \in \mathcal{X}$; so $\mathcal{X} \subseteq C$. If $\cup \mathcal{X}=\emptyset$, as $\emptyset \in C$, we have $(p, \cup \mathcal{X})=(p, \emptyset) \in \mathcal{R}$. Assume thus that $\cup \mathcal{X} \neq \emptyset$ and let $\mathcal{Y}=\mathcal{X} \backslash\{\emptyset\}$, so $\cup \mathcal{Y}=\cup \mathcal{X}$. For $S \in \mathcal{Y}$, as $S \neq \emptyset$ and $(p, S) \in \Omega(E)$, we have $p \in S$; hence $p \in \cap \mathcal{Y}$; but $\mathcal{Y} \subseteq \mathcal{C}$, so $\bigcup \mathcal{Y} \in C$. As $p \in \bigcup \mathcal{Y}$, we have $(p, \cup \mathcal{X})=(p, \cup \mathcal{Y}) \in \mathcal{R}$. Hence $\mathcal{R}$ satisfies the union property.

Now let $(p, S) \in \mathcal{R}, q \in S$ and $(q, T) \in \mathcal{R}$. If $T=\emptyset$, then $(p, S \cup T)=(p, S) \in \mathcal{R}$. If $T \neq \emptyset$, as $(q, T) \in \mathcal{R}$, we get $q \in T$; since $q \in S, S \neq \emptyset$, and as $(p, S) \in \mathcal{R}$, we have $p \in S$. Since $S, T \in C$ and $q \in S \cap T$, we get $S \cup T \in C$, and as $p \in S$, we have $p \in S \cup T$, so $(p, S \cup T) \in \mathcal{R}$. Hence $\mathcal{R}$ satisfies the transitivity property. Therefore $\mathcal{R}$ will be a reach.

Finally, if $C$ is a connection, then for any $p \in E,\{p\} \in C$, thus $(p,\{p\}) \in \mathcal{R}$, hence the reach $\mathcal{R}$ is full.

In the Tankyevych reach, we note that given $(p, P),(q, Q) \in$ $\mathcal{T}_{(V, A)}$ such that $q \in P$ and $p \in Q$, there is in $P$ a directed path from $p$ to $q$, and in $Q$ a directed path from $q$ to $p$, then the union of these two paths is strongly connected. This property will not extend to an arbitrary reach: for $(p, P),(q, Q) \in \mathcal{R}$ with $q \in P$ and $p \in Q$, it does not follow that there exists some $S \in \operatorname{Sym}(\mathcal{R})$ with $p, q \in S$. For example, take $E$ of size at least 3, choose two distinct $p, q \in E$, and let $\mathcal{R}=\mathrm{O}(E) \cup\{(p, E),(q, E)\}$; then $\mathcal{R}$ is a reach where $(p, E),(q, E) \in \mathcal{R}$ with $p, q \in E$, but $\operatorname{Sym}(\mathcal{R})=\{\emptyset\}$.

\section{The lattice of reaches}

We will analyse the inclusion order on reaches, and the complete lattice structure that it induces. This will allow us to describe the reach generated by a subset of $\mathcal{R} \subseteq E \times \mathcal{P}(E)$. Our approach is very similar to that of Section 2.4 in (Ronse, 2008). Let us write $\mathfrak{R}(E), \mathfrak{R} \mathrm{f}(E), \mathfrak{R s}(E)$ and $\mathfrak{R f s}(E)$ respectively for the set of all reaches, full reaches, symmetrical reaches and symmetrical full reaches on $E$.

Theorem 10. The four sets $\mathfrak{R}(E), \mathfrak{R} \mathrm{f}(E), \mathfrak{R s}(E)$ and $\mathfrak{R f s}(E)$, ordered by inclusion, are complete lattices, with $\Omega(E)$ as greatest element, and the non-empty infimum operation is given by the intersection. The least element of $\mathfrak{R}(E)$ and of $\mathfrak{R}(E)$ is $\mathrm{O}(E)$, while the least element of $\mathfrak{R}(E)$ and of $\mathfrak{R s}(E)$ is $\Phi(E)$. More precisely:

1. The greatest reach on $E$ is $\Omega(E)$, it is symmetrical and full, and its system of point openings $\left(\omega_{p}, p \in E\right)$ is given by $\omega_{p}(X)=X$ if $p \in X$, and $\omega_{p}(X)=\emptyset$ if $p \notin X$. The least reach on $E$ is $\mathrm{O}(E)$, it is symmetrical, and its system of point openings $\left(o_{p}, p \in E\right)$ is given by $o_{p}(X)=\emptyset$ for all $p \in E$ and $X \in \mathcal{P}(E)$. The least full reach on $E$ is $\Phi(E)$, it is symmetrical, and its system of point openings $\left(\phi_{p}, p \in E\right)$ is given by $\phi_{p}(X)=\{p\}$ if $p \in X$, and $\phi_{p}(X)=\emptyset$ if $p \notin X$.

2. Given two reaches $\mathcal{R}$ and $\mathcal{R}^{\prime}$ on $E$ with systems of point openings $\left(\gamma_{p}, p \in E\right)$ and $\left(\gamma_{p}^{\prime}, p \in E\right)$, we have $\mathcal{R} \subseteq \mathcal{R}^{\prime}$ if and only if for every $p \in E$ we have $\operatorname{Inv}\left(\gamma_{p}\right) \subseteq \operatorname{lnv}\left(\gamma_{p}^{\prime}\right)$, in other words $\gamma_{p} \leq \gamma_{p}^{\prime}$.

3. Given a non-empty family $\mathcal{R}_{i}(i \in I, I \neq \emptyset)$ of reaches on $E$, with corresponding systems of point openings $\left(\gamma_{p}^{i}, p \in E\right)$, then $\bigcap_{i \in I} \mathcal{R}_{i}$ is a reach on $E$, its system of point openings $\left(\gamma_{p}, p \in E\right)$ satisfies

$$
\forall p \in E, \quad \operatorname{lnv}\left(\gamma_{p}\right)=\bigcap_{i \in I} \operatorname{Inv}\left(\gamma_{p}^{i}\right)
$$

in other words $\gamma_{p}$ is the infimum of the $\gamma_{p}^{i}, i \in I$, in the lattice of openings on $\mathcal{P}(E)$. If the reaches $\mathcal{R}_{i}$ are all full, then $\bigcap_{i \in I} \mathcal{R}_{i}$ is also full. We have:

$$
\operatorname{Sym}\left(\bigcap_{i \in I} \mathcal{R}_{i}\right)=\bigcap_{i \in I} \operatorname{Sym}\left(\mathcal{R}_{i}\right) \text {. }
$$

If the reaches $\mathcal{R}_{i}$ are all symmetrical, then $\bigcap_{i \in I} \mathcal{R}_{i}$ is also symmetrical.

Proof. 1. The union and membership properties are satisfied by $\mathrm{O}(E)$ and $\Omega(E)$. Now for the transitivity property, the condition in Definition 2 states $q \in S$, so $S \neq \emptyset$; thus the condition cannot apply in $\mathrm{O}(E)$, while in $\Omega(E)$ we must have $(p, S) \in \Psi(E)$, that is $p \in S$, hence $p \in S \cup T$, so $(p, S \cup T) \in \Psi(E) \subseteq \Omega(E)$. Hence $\mathrm{O}(E)$ and $\Omega(E)$ are reaches; as $\Sigma(E) \subseteq \Omega(E), \Omega(E)$ is full.

For any reach $\mathcal{R}$, the union property gives $\mathrm{O}(E) \subseteq \mathcal{R}$, while the membership property gives $\mathcal{R} \subseteq \Omega(E)$. Thus $\mathrm{O}(E)$ and $\Omega(E)$ are the least and greatest reaches. By Proposition 6 , the least full reach will be $\mathrm{O}(E) \cup \Sigma(E)=\Phi(E)$. It is easily seen that $\mathrm{O}(E), \Phi(E)$ and $\Omega(E)$ are symmetrical, see Definition 6 .

To describe the systems of point openings $\left(o_{p}, p \in E\right)$ and ( $\left.\omega_{p}, p \in E\right)$, we apply (2): the only $A \subseteq X$ with $(p, A) \in \mathrm{O}(E)$ is $A=\emptyset$; now the $A \subseteq X$ with $(p, A) \in \Omega(E)$ are only $A=\emptyset$ for $p \notin X$, but all $A \in \mathcal{P}(X)$ for $p \in X$. Then the expression for $\left(\phi_{p}, p \in E\right)$ is derived from the one for $\left(o_{p}, p \in E\right)$, thanks to (5).

2. By (3), for any $p \in E$ and $S \in \mathcal{P}(E),(p, S) \in \mathcal{R} \Leftrightarrow S \in$ $\operatorname{lnv}\left(\gamma_{p}\right)$ and $(p, S) \in \mathcal{R}^{\prime} \Leftrightarrow S \in \operatorname{Inv}\left(\gamma_{p}^{\prime}\right)$. Thus

$$
\mathcal{R} \subseteq \mathcal{R}^{\prime} \Leftrightarrow\left[\forall p \in E, \operatorname{Inv}\left(\gamma_{p}\right) \subseteq \operatorname{Inv}\left(\gamma_{p}^{\prime}\right)\right]
$$

By the isomorphism between the lattices of openings and of families closed under union, this means that $\forall p \in E, \gamma_{p} \leq \gamma_{p}^{\prime}$.

3. It is easily checked that the union, transitivity, membership and point properties are all preserved by a non-empty intersection of families $\mathcal{R}_{i}$. (NB. The membership property is not satisfied by an empty intersection of families.) Thus $\bigcap_{i \in I} \mathcal{R}_{i}$ is a reach, and if the $\mathcal{R}_{i}$ are all full, then $\bigcap_{i \in I} \mathcal{R}_{i}$ is also full. 
By (3), for any $p \in E$ and $S \in \mathcal{P}(E)$,

$$
\begin{gathered}
S \in \operatorname{Inv}\left(\gamma_{p}\right) \Leftrightarrow(p, S) \in \bigcap_{i \in I} \mathcal{R}_{i} \Leftrightarrow\left[\forall i \in I,(p, S) \in \mathcal{R}_{i}\right] \\
\Leftrightarrow\left[\forall i \in I, S \in \operatorname{Inv}\left(\gamma_{p}^{i}\right)\right] \Leftrightarrow S \in \bigcap_{i \in I} \operatorname{Inv}\left(\gamma_{p}^{i}\right),
\end{gathered}
$$

so $\operatorname{lnv}\left(\gamma_{p}\right)=\bigcap_{i \in I} \operatorname{lnv}\left(\gamma_{p}^{i}\right)$ for all $p \in E$.

Apply (6): for any $S \in \mathcal{P}(E)$, we have $S \in \operatorname{Sym}\left(\bigcap_{i \in I} \mathcal{R}_{i}\right)$ iff $\forall q \in S,(q, S) \in \bigcap_{i \in I} \mathcal{R}_{i}$, iff $\forall i \in I, \forall q \in S,(q, S) \in \mathcal{R}_{i}$, iff $\forall i \in I, S \in \operatorname{Sym}\left(\mathcal{R}_{i}\right)$, iff $S \in \bigcap_{i \in I} \operatorname{Sym}\left(\mathcal{R}_{i}\right)$. Thus $\operatorname{Sym}\left(\bigcap_{i \in I} \mathcal{R}_{i}\right)=\bigcap_{i \in I} \operatorname{Sym}\left(\mathcal{R}_{i}\right)$. If all $\mathcal{R}_{i}$ are symmetrical, then for $(p, S) \in \bigcap_{i \in I} \mathcal{R}_{i}$ we have $\forall i \in I,(p, S) \in \mathcal{R}_{i}$, and the symmetry of $\mathcal{R}_{i}$ gives $S \in \operatorname{Sym}\left(\mathcal{R}_{i}\right)$ (see Definition 6), hence $S \in \bigcap_{i \in I} \operatorname{Sym}\left(\mathcal{R}_{i}\right)=\operatorname{Sym}\left(\bigcap_{i \in I} \mathcal{R}_{i}\right)$, therefore $\bigcap_{i \in I} \mathcal{R}_{i}$ is symmetrical.

Since $\mathfrak{R}(E), \mathfrak{R f}(E), \mathfrak{R s}(E)$ and $\mathfrak{R f s}(E)$ are closed under non-empty intersection and have a common greatest element $\Omega(E)$, they constitute complete lattices.

One can consider a hierarchy of full reaches, that is, a chain $\Phi(E)=\mathcal{R}_{0} \subset \cdots \subset \mathcal{R}_{n}=\Omega(E)$ of full reaches (Tankyevych et al., 2013; Perret et al., 2014), with corresponding chains of point openings $\phi_{p}=\gamma_{p}^{0} \leq \cdots \leq \gamma_{p}^{n}=\omega_{p}, p \in E$; for $p \in X \subseteq E$ we have $\gamma_{p}^{i}(X) \subseteq \gamma_{p}^{i+1}(X)(i=0, \ldots, n-1)$, but there can be several $q \in X$ with distinct $\gamma_{q}^{i+1}(X)$ such that $\gamma_{p}^{i}(X) \subseteq \gamma_{q}^{i+1}(X)$ : thus semi-connected components in a hierarchy of full reaches do not make a rooted tree (Perret et al., 2014); this contrasts with hierarchies of connections, where we have a rooted tree, since $\gamma_{p}^{i+1}(X)$ is the only connected component of $X$ at level $i+1$ containing $\gamma_{p}^{i}(X)$.

Given $\mathcal{B} \subseteq \Omega(E)$, there is at least one reach containing $\mathcal{B}$, namely $\Omega(E)$. Thus the intersection of all reaches containing $\mathcal{B}$ will be the least reach containing $\mathcal{B}$, we call it the reach generated by $\mathcal{B}$ and write it $\operatorname{Reach}(\mathcal{B})$. Since every reach contains $\mathrm{O}(E)$, the elements of $\mathrm{O}(E)$ are useless for the generation of a reach, that is, $\operatorname{Reach}(\mathcal{B})=\operatorname{Reach}(\mathcal{B} \backslash \mathrm{O}(E))$. Hence we can without loss of generality assume that $\mathcal{B} \subseteq \Psi(E)$.

The same can be said for full reaches, the intersection of all full reaches containing $\mathcal{B}$ will be the full reach generated by $\mathcal{B}$; we will not introduce any notation for it, since by Proposition 6 it is in fact $\operatorname{Reach}(\mathcal{B}) \cup \Sigma(E)$.

Note that when $\mathcal{B} \subseteq E \times \mathcal{P}(E)$ but $\mathcal{B} \nsubseteq \Omega(E)$, there is no reach containing $\mathcal{B}$, because the membership property is not satisfied: there is some $(p, S) \in \mathcal{B}$ with $S \neq \emptyset$ and $p \notin S$.

We will now introduce the analogue of the notion of chaining used in the generation of (partial) connections (Ronse, 1998, 2008). We call it linking; it will allow us to describe $\operatorname{Reach}(\mathcal{B})$. Note that for $S \subseteq E, \Psi(S)=\Psi(E) \cap(S \times \mathcal{P}(S))$.

Definition 7. (See Figure 2.) Let $\mathcal{B} \subseteq \Psi(E)$.

1. Given two points $p, q \in E$, we say that $\mathcal{B}$ joins $p$ to $q$ if for some $n \geq 0$ there are $\left(p_{0}, S_{0}\right), \ldots,\left(p_{n}, S_{n}\right) \in \mathcal{B}$ such that $p=p_{0}, q \in S_{n}$ and $p_{i} \in S_{i-1}$ for $i=1, \ldots, n$.

2. Given $(p, S) \in \Omega(E)$, we say that $\mathcal{B}$ links $p$ to $S$ if $\mathcal{B} \subseteq$ $S \times \mathcal{P}(S)($ that is, $\mathcal{B} \subseteq \Psi(S))$ and for every $x \in S, \mathcal{B}$ joins $p$ to $x$.
Note that trivially $\emptyset$ (subset of $\Omega(E)$ ) links $p$ to $\emptyset$ (subset of $E$ ). It is easily seen that the property of joining is transitive: if $\mathcal{B}$ joins $p$ to $q$ and $q$ to $r$, then it joins $p$ to $r$.

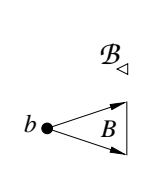

(a)

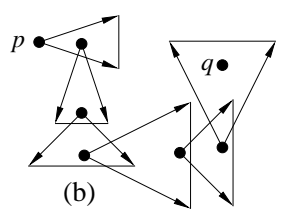

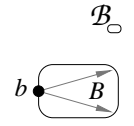

(c)

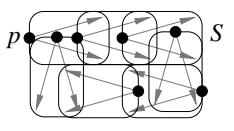

(d)
Figure 2: Let $\mathcal{B}_{\triangleleft} \subseteq \Psi(E)$, where (a) for each $(b, B) \in \mathcal{B}_{\triangleleft}, B$ is a triangle and $b$ is shown as a black dot on its corner, and the two arrows pointing out of it indicate that $B$ is reachable from $b$. Then (b) $\mathcal{B}_{\triangleleft}$ joins $p$ to $q$ through a sequence of 6 elements of $\mathcal{B}_{\triangleleft}$. Let $\mathcal{B}_{\odot} \subseteq \Psi(E)$, where (c) for each $(b, B) \in \mathcal{B}_{\odot}, B$ is a rounded rectangle. Then (d) $\mathcal{B}_{\odot}$ links $p$ to the set $S$ (the big rectangle with rounded corners), using 7 elements of $\mathcal{B}_{\odot}$.

Proposition 11. For any $\mathcal{B} \subseteq \Psi(E)$, Reach $(\mathcal{B})$ is the set of all $(p, S) \in \Omega(E)$ such that $\mathcal{B} \cap(S \times \mathcal{P}(S))=\mathcal{B} \cap \Psi(S)$ links $p$ to $S$. In other words, for $(p, S) \in \Omega(E)$, we have $(p, S) \in$ $\operatorname{Reach}(\mathcal{B})$ if and only if either $S=\emptyset$ or for any $q \in S$ there are $p_{0}, \ldots, p_{n} \in S$ and $S_{0}, \ldots, S_{n} \in \mathcal{P}(S)(n \geq 0)$ such that $\left(p_{0}, S_{0}\right), \ldots,\left(p_{n}, S_{n}\right) \in \mathcal{B}, p=p_{0}, q \in S_{n}$ and $p_{i} \in S_{i-1}$ for $i=1, \ldots, n$.

Proof. As $\mathcal{B} \subseteq \Psi(E)$, for $S \in \mathcal{P}(E)$ we have

$$
\mathcal{B} \cap \Psi(S)=\mathcal{B} \cap(S \times \mathcal{P}(S))=\{(q, T) \in \mathcal{B} \mid q \in S, T \subseteq S\}
$$

Let $\mathcal{D}$ be the set of all $(p, S) \in \Omega(E)$ such that $\mathcal{B} \cap \Psi(S)$ links $p$ to $S$. Let us show that $\mathcal{D}$ is a reach. Let $p \in E$. As $\mathcal{B} \cap \Psi(\emptyset)=\emptyset$ trivially links $p$ to $\emptyset,(p, \emptyset) \in \mathcal{D}$. Now let $\mathcal{X} \subseteq \mathcal{P}(E)$ such that $\cup \mathcal{X} \neq \emptyset$ and $(p, S) \in \mathcal{D}$ for every $S \in \mathcal{X}$. For any $x \in \cup \mathcal{X}$ there is some $S \in \mathcal{X}$ such that $x \in S$, then $\mathcal{B} \cap \Psi(S)$ links $p$ to $S$, so it joins $p$ to $x$; now $S \subseteq \cup \mathcal{X}$, so $\mathcal{B} \cap \Psi(S) \subseteq \mathcal{B} \cap \Psi(\cup \mathcal{X})$, thus $\mathcal{B} \cap \Psi(\cup \mathcal{X})$ joins $p$ to any such $x$, so it links $p$ to $\bigcup \mathcal{X}$. Hence $(p, \cup \mathcal{X}) \in \mathcal{D}$, and $\mathcal{D}$ satisfies the union property.

Let $(p, S) \in \mathcal{D}, q \in S$ and $(q, T) \in \mathcal{D}$; thus $\mathcal{B} \cap \Psi(S)$ links $p$ to $S$, and $\mathcal{B} \cap \Psi(T)$ links $q$ to $T$. As $\mathcal{B} \cap \Psi(S), \mathcal{B} \cap \Psi(T) \subseteq$ $\mathcal{B} \cap \Psi(S \cup T), \mathcal{B} \cap \Psi(S \cup T)$ joins $p$ to any $x \in S$ and joins $q$ to any $y \in T$; as $\mathcal{B} \cap \Psi(S \cup T)$ joins $p$ to $q$ and $q$ to $y$, it joins $p$ to $y$. Therefore $\mathcal{B} \cap \Psi(S \cup T)$ joins $p$ to any point of $S \cup T$, so it links $p$ to $S \cup T$, and the transitivity property is satisfied.

Let $(p, S) \in \mathcal{D}$ such that $S \neq \emptyset$, and take $x \in S$; then $\mathcal{B} \cap \Psi(S)$ joins $p$ to $x$, thus we have $\left(p_{0}, S_{0}\right) \in \mathcal{B} \cap \Psi(S)$ with $p=p_{0}$ and either $p_{1} \in S_{0}$ (if $n>0$ ) or $x \in S_{0}$ (if $n=0$ ); so $S_{0} \neq \emptyset$, and as $\left(p_{0}, S_{0}\right) \in \mathcal{B} \cap \Psi(S), p=p_{0} \in S_{0} \subseteq S$. Hence $\mathcal{D}$ satisfies the membership property. Therefore $\mathcal{D}$ is a reach.

For $(p, S) \in \mathcal{B},\{(p, S)\}$ joins $p$ to every $x \in S$ (take $n=0$ and $\left.S_{0}=S\right)$, and $\{(p, S)\} \subseteq \mathcal{B} \cap \Psi(S)$, thus $\mathcal{B} \cap \Psi(S)$ links $p$ to $S$. Therefore $\mathcal{B} \subseteq \mathcal{D}$.

Let $\mathcal{R}$ be a reach containing $\mathcal{B}$. We show that $\mathcal{D} \subseteq \mathcal{R}$. Let $(p, S) \in \mathcal{D}$; thus $\mathcal{B} \cap \Psi(S)$ links $p$ to $S$. If $S=\emptyset$, then $(p, S)=(p, \emptyset) \in \mathcal{R}$ by the union property; assume thus that $S \neq \emptyset$. Take any $x \in S$, so $\mathcal{B} \cap \Psi(S)$ joins $p$ to $x$ : there are $\left(p_{0}, S_{0}\right), \ldots,\left(p_{n}, S_{n}\right) \in \mathcal{B} \cap \Psi(S)(n \geq 0)$ such that $p=p_{0}, x \in S_{n}$ and $p_{i} \in S_{i-1}$ for $i=1, \ldots, n$. Thus $S_{0}, \ldots, S_{n} \subseteq S$, so $S_{0} \cup \cdots \cup S_{n} \subseteq S$. By induction on 
$j=0, \ldots, n$ we have $\left(p, S_{0} \cup \cdots \cup S_{j}\right) \in \mathcal{R}$ : (a) for $j=0$ we have $\left(p, S_{0}\right)=\left(p_{0}, S_{0}\right) \in \mathcal{B} \subseteq \mathcal{R}$; (b) if the result holds for $j$, then it holds for $j+1$ : as $\left(p, S_{0} \cup \cdots \cup S_{j}\right) \in \mathcal{R}, p_{j+1} \in S_{j}$ and $\left(p_{j+1}, S_{j+1}\right) \in \mathcal{B} \subseteq \mathcal{R}$, by the transitivity property we have $\left(p, S_{0} \cup \cdots \cup S_{j} \cup S_{j+1}\right) \in \mathcal{R}$. Thus $\left(p, S_{0} \cup \cdots \cup S_{n}\right) \in \mathcal{R}$, with $x \in S_{0} \cup \cdots \cup S_{n} \subseteq S$. Hence for any $x \in S$ there exists $S_{x} \subseteq S$ such that $x \in S_{x}$ and $\left(p, S_{x}\right) \in \mathcal{R}$; so $S=\bigcup_{x \in S} S_{x}$ and by the union property, $(p, S)=\left(p, \bigcup_{x \in S} S_{x}\right) \in \mathcal{R}$. Therefore $\mathcal{D} \subseteq \mathcal{R}$.

Since $\mathcal{D}$ is a reach containing $\mathcal{B}$, and every reach containing $\mathcal{B}$ must contain $\mathcal{D}$, it follows that $\mathcal{D}$ is the least reach containing $\mathcal{B}$, that is, $\operatorname{Reach}(\mathcal{B})$.

For example, the inheritance reach, see Figure 1, is the full reach $\operatorname{Reach}(\mathcal{B}) \cup \Sigma(V)$ generated by the set $\mathcal{B}$ consisting of all pairs $(p, S)$, where $p$ is a non-leaf vertex and $S$ is the set made of $p$ and all its children vertices. The Tankyevych reach $\mathcal{T}_{(V, A)}$ is the full reach generated by all $(p,\{p, q\})$ for $p, q \in V$ such that $(p, q) \in A$. For a quasi-order $\sqsubseteq$ on $V, \mathcal{A}_{(V, \subseteq, A)}$ is the full reach generated by all such $(p,\{p, q\}),(p, q) \in A$, with the further restriction that $p \sqsubseteq q$.

Linking allows us to construct new reaches. We illustrate this first with some new examples defined on arc-weighted directed graphs. Let $(V, A)$ be a simple directed graph, and assume that a strictly positive weight function is defined on $A$ : to every arc $(p, q) \in A$ is associated a weight $w(p, q)>0$. For each vertex $p \in V$, define the ingoing neighbourhood $N^{-}(p)$ and outgoing neighbourhood $N^{+}(p)$ :

$N^{-}(p)=\{q \in V \mid(q, p) \in A\}, \quad N^{+}(p)=\{q \in V \mid(p, q) \in A\}$.

For every vertex $p \in V$ and real number $t \geq 0$, let

$$
W(p, t)=\{p\} \cup\left\{q \in N^{+}(p) \mid w(p, q)<t\right\}
$$

be the set made of $p$ and all outgoing neighbours joined by an arc of weight $<t$. Note that $W(p, 0)=\{p\}$ (since all $w(p, q)>0)$, and for a family of values $t_{i} \geq 0, i \in I$, we have $W\left(p, \sup _{i \in I} t_{i}\right)=\bigcup_{i \in I} W\left(p, t_{i}\right)$. For any $t \geq 0$, let $\mathcal{W}_{t}$ be the family of all $(p, W(p, t))$ for $p \in V$. We illustrate in Figure 3 $\operatorname{Reach}\left(\mathcal{W}_{t}\right)$ for a rooted tree.

Now let $\mathcal{D}$ be the set of all $(p,\{p, q\})$ for $p, q \in V$, such that $(p, q) \in A$ and for every $r \in N^{-}(p), w(r, p) \geq w(p, q)$. The full reach Reach $(\mathcal{D}) \cup \Sigma(V)$ generated by it will be included in the Tankyevych reach $\mathcal{T}_{(V, A)}$. Given $(p, S) \in \operatorname{Reach}(\mathcal{D}) \cup \Sigma(V)$, every $x \in S$ can be reached from $p$ by a path whose successive arcs have decreasing weights; this condition is necessary, but generally not sufficient; when $(V, A)$ is a rooted tree, the necessary and sufficient condition for $(p, S) \in \operatorname{Reach}(\mathcal{D}) \cup \Sigma(V)$ is that $S \subseteq\{p\}$ or $S$ is the set of vertices of a subtree with root $p$, such that the weights of the arcs decrease on any directed path in $S$, and either $p$ is the root of $V$, or the unique $r \in N^{-}(p)$ (the parent vertex of $p$ ) satisfies $w(r, p) \geq w(p, q)$ for all $q \in N^{+}(p) \cap S$ (i.e., for all children nodes $q$ of $p$ inside $S$ ).

Our next example considers a structuring element in a digital space. Let $E=\mathbf{Z}^{n}$, with origin $o=(0, \ldots, 0)$, and take $B \subseteq E$ such that $o \in B$. We set $\mathcal{B}=\left\{\left(p, B_{p}\right) \mid p \in E\right\}$, where $B_{p}=$ $\{b+p \mid b \in B\}$ is the translate of $B$ by $p$. In Figure 4 we compare point openings in $\operatorname{Reach}(\mathcal{B})$ with the connected components in the partial connection generated by the $B_{p}, p \in E$.
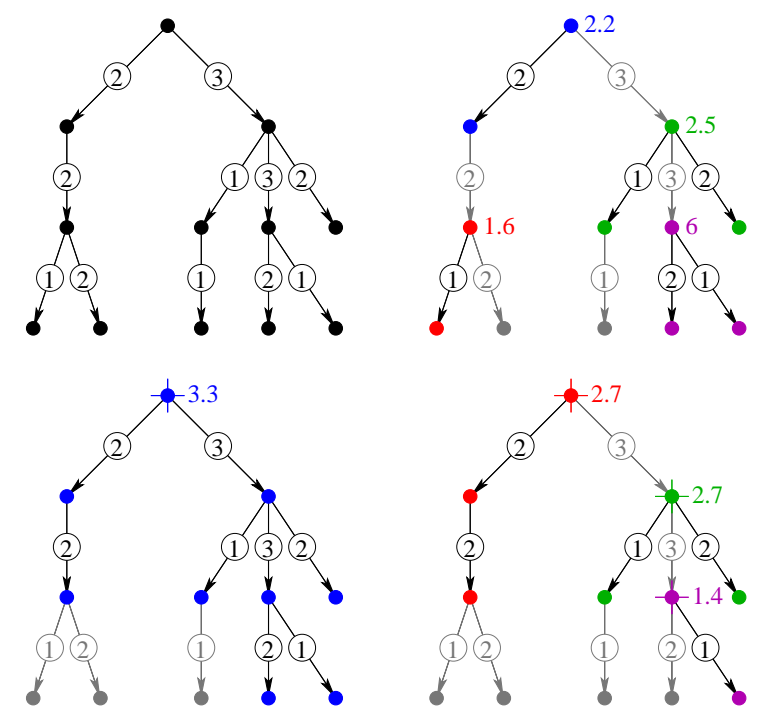

Figure 3: Top left: a rooted tree with integer edge weights. Top right: 4 sets $W(p, t)$ of vertices, for $t=2.2$ (in blue), $t=2.5$ (in green), $t=1.6$ (in red) and $t=6$ (in magenta). Bottom left: the blue marker $p$ indicated by a cross and the reached set $S$ of blue vertices constitute an element $(p, S)$ of Reach $\left(\mathcal{W}_{3.3}\right)$. Bottom right: two elements of Reach $\left(\mathcal{W}_{2.7}\right)$ (in red and in green), and an element of Reach $\left(\mathcal{W}_{1.4}\right)$ (in magenta); in each the marker is crossed.
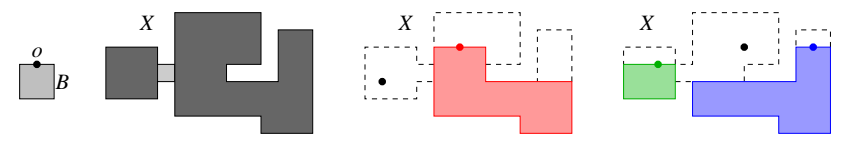

Figure 4: Let $E=\mathbf{Z}^{2}$. Left: we show the pair $(o, B)$, with $o \in B$, then the set $X$ decomposed, in the partial connection generated by the translates of $B$, into two connected components (in dark grey) and a residual (in light grey). Middle and right: for several marker points $p \in E$ we show (in the same colour) the opening $\gamma_{p}(X)$; for the black markers $p, \gamma_{p}(X)=\emptyset$. Since $B$ is under $o$, the joining process cannot move upwards.

\section{Discussion and conclusion}

In medical imaging, a vascular network can be represented by a directed graph. The arcs correspond to the branches of the network, and they are oriented according to the direction of the blood flow; the vertices correspond to the origin of the network, bifurcations, blood vessel terminations, or locations of interest such as stenoses. For several arterial networks, such as the coronary arteries originating from the aorta (Bouraoui et al., 2010), this graph is a rooted tree, see Figure 5.

The arcs can be weighted by various measurements on the branches (such as width, length, etc), they can also be labelled according to the anatomical labels of the branches. For instance, if the arc weight corresponds to the width of the vessel branch, some reaches described above can be useful for selecting specific connected subsets of the network: Reach $\left(\mathcal{W}_{t}\right) \cup$ $\Sigma(V)$ will select those having blood vessels narrower than $t$, while Reach $(\mathcal{D}) \cup \Sigma(V)$ will select those where the width of blood vessels decreases in the direction of blood flow.

Tankyevych et al. (2013) applied semi-connected sets (i.e., the Tankyevych reach) to the segmentation of vascular structures. Perret et al. (2014) applied them to the segmentation of 

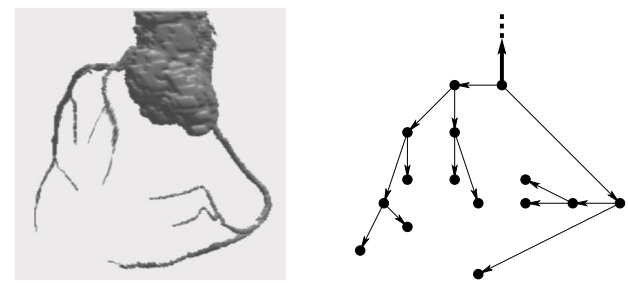

Figure 5: Left: from Bouraoui et al. (2010), segmentation of the start of the aorta (directed upwards) and of the two coronary arteries. Right: the corresponding directed graph (the thick arc denotes the aorta).

neurite and miocardium images. Other reaches could also be applied.

A theoretical perspective is the design of new reaches. Several works, in particular (Ouzounis and Wilkinson, 2007; Ronse, 1998, 2008; Ronse and Serra, 2010; Serra, 1988), studied second generation connectivity, that is, methods for generating new (partial) connections from existing ones. It could be possible to generalize such methods to the case of reaches.

In morphological image filtering, connectivity intervenes in the design of connected operators, that is, operators that can merge objects, but do not deform their contours (Ronse and Serra, 2010; Salembier, 2010; Salembier and Wilkinson, 2009; Serra et al., 2010). An important tool in the design of such operators is the operation of geodesic reconstruction. The links between connections and geodesic reconstruction have been studied in the abstract framework of complete lattices (Braga-Neto and Goutsias, 2002; Ronse and Serra, 2001); in particular, the latter paper axiomatized the notion of geodesic reconstruction in a way that generalizes usual geodesic reconstructions by dilation or by erosion.

According to our preliminary investigations, given two sets $R, S \in \mathcal{P}(E)$ such that $R \subseteq S$ and a full reach $\mathcal{R}$ corresponding to the system of point openings $\left(\gamma_{p}, p \in E\right)$ satisfying (T), (M) / $\left(\mathrm{M}^{\prime}\right)$ and $(\mathrm{P}) /\left(\mathrm{P}^{\prime}\right)$, the operation $(S, R) \mapsto \bigcup_{p \in R} \gamma_{p}(S)$ is a geodesic reconstruction in the sense of Ronse and Serra (2001), and the Tankyevych reach corresponds to the geodesic reconstruction obtained by iteration of a conditional dilation on the marker $R$ inside the mask $S$. This topic, as well as the possible extension of the theory to arbitrary complete lattices, will be studied in further papers. In particular, the oriented geodesic reconstruction for grey-level images, introduced by Vincent (1998), could be analysed from a topological point of view.

We conclude. We have succeeded in establishing a theory of oriented connected structures, thanks to the new concepts of a reach and of a full reach. Partial connections and connections arise under the special condition of symmetry. As in the theory of connections and partial connections (Serra, 1988; Ronse, 2008), we gave an alternate characterization in terms of a system of point openings satisfying some properties, and we could describe the complete lattice of reaches (and the one of full reaches); in particular we could show how to generate a reach from a family of pairs $(p, S) \in E \times \mathcal{P}(E)$ by a process similar to chaining.

We indicated possible applications of our theory to the study of vascular networks in medical imaging. Other oriented and connected structures could be studied with our framework.

\section{References}

Bertrand, G., Couprie, M., Cousty, J., Najman, L., 2010. Watersheds in discrete spaces, in: Najman, L., Talbot, H. (Eds.), Mathematical morphology: From Theory to Applications. ISTE / J. Wiley \& Sons. chapter 3, pp. 81-107.

Bouraoui, B., Ronse, C., Baruthio, J., Passat, N., Germain, P., 2010. 3d segmentation of coronary arteries based on advanced mathematical morphology techniques. Computerized Medical Imaging and Graphics 34, 377-387. doi:10.1016/j.compmedimag.2010.01.001.

Braga-Neto, U., Goutsias, J., 2002. Connectivity on complete lattices: new results. Computer Vision and Image Understanding 85, 22-53.

Cousty, J., Bertrand, G., Couprie, M., Najman, L., 2014. Collapses and watersheds in pseudomanifolds of arbitrary dimension. Journal of Mathematical Imaging and Vision to appear. doi:10.1007/s10851-014-0498-Z.

Heijmans, H., 1994. Morphological Image Operators. Advances in Electronics and Electron Physics Series, Academic Press, Boston.

Ouzounis, G., Wilkinson, M., 2007. Mask-based second-generation connectivity and attribute filters. IEEE Transactions on Pattern Analysis and Machine Intelligence 29, 990-1004. doi:10.1109/TPAMI.2007.1045.

Perret, B., Cousty, J., Tankyevych, O., Talbot, H., Passat, N., 2014. Directed connected operators: asymmetric hierarchies for image filtering and segmentation. Submitted

Ronse, C., 1998. Set-theoretical algebraic approaches to connectivity in continuous or digital spaces. Journal of Mathematical Imaging and Vision 8, 41-58. doi:10.1023/A:1008210216583.

Ronse, C., 2008. Partial partitions, partial connections and connective segmentation. Journal of Mathematical Imaging and Vision 32, 97-125. doi:10.1007/s10851-008-0090-5.

Ronse, C., Serra, J., 2001. Geodesy and connectivity in lattices. Fundamenta Informaticae 46, 349-395.

Ronse, C., Serra, J., 2010. Algebraic foundations of morphology, in: Najman, L., Talbot, H. (Eds.), Mathematical morphology: From Theory to Applications. ISTE / J. Wiley \& Sons. chapter 2, pp. 35-80.

Salembier, P., 2010. Connected operators based on tree pruning strategies, in: Najman, L., Talbot, H. (Eds.), Mathematical morphology: From Theory to Applications. ISTE / J. Wiley \& Sons. chapter 7, pp. 179-198.

Salembier, P., Wilkinson, M.H.F., 2009. Connected operators: A review of region-based morphological image processing techniques. IEEE Signal Processing Magazine 26, 136-157.

Serra, J., 1988. Mathematical morphology for Boolean lattices, in: Serra, J. (Ed.), Image Analysis and Mathematical Morphology, II: Theoretical Advances. Academic Press, London. chapter 2, pp. 37-58.

Serra, J., 2011. Hierarchies and optima, in: Debled-Rennesson, I., Domenjoud, E., Kerautret, B., Even, P. (Eds.), DGCI 2011, 16th International Conference on Discrete Geometry for Computer Imagery. Springer-Verlag, Berlin. volume 6607 of Lecture Notes in Computer Science, pp. 35-46.

Serra, J., Vachier, C., Meyer, F., 2010. Levelings, in: Najman, L., Talbot, H. (Eds.), Mathematical morphology: From Theory to Applications. ISTE / J. Wiley \& Sons. chapter 8, pp. 199-228.

Tankyevych, O., Talbot, H., Passat, N., 2013. Semi-connections and hierarchies, in: Luengo Hendriks, C.L., Borgefors, G., Strand, R. (Eds.), Mathematical Morphology and Its Applications to Signal and Image Processing. Springer, Berlin. volume 7883 of Lecture Notes in Computer Science, pp. 159-170. doi:10.1007/978-3-642-38294-9_14.

Vincent, L., 1998. Minimal path algorithms for the robust detection of linear features in gray images. Computational Imaging and Vision 12, 331-338. 